\title{
GIANT RETINAL TEAR AND RETINAL DETACHMENT WITH UNDERLYING RETINITIS PIGMENTOSA AND HEARING LOSS
}

\author{
ROBERT Y. KIM ${ }^{1,2}$, STEVEN D. SCHWARTZ ${ }^{1,3}$, JOHN R. HECKENLIVELY ${ }^{3}$, \\ ZDENEK J. GREGOR ${ }^{1}$ and ROBERT J. COOLING ${ }^{1}$ \\ London, San Francisco and Los Angeles
}

\begin{abstract}
SUMMARY
Few retinal detachments have been described in patients with retinitis pigmentosa and allied retinal disorders, with only two cases (both with hearing loss) reported in association with giant retinal tears. To further characterise clinical characteristics of giant retinal tear associated with retinitis pigmentosa, we reviewed the course of four eyes of three patients. Unexpectedly, all three individuals also suffered from congenital sensorineural hearing loss. One suffered from associated myopathy. Despite aggressive surgical management, three of the four eyes became blind. The diagnosis of retinal detachment preceded the diagnosis of photoreceptor dystrophy in two of the three patients. To date, giant retinal tears occurring with underlying retinitis pigmentosa have been described in five young individuals, all of whom had associated congenital sensorineural hearing loss.
\end{abstract}

Few cases of retinal detachment in patients with retinitis pigmentosa and allied diseases have been reported, ${ }^{1-7}$ with several of them associated with factors such as trauma ${ }^{3,5}$ or previous cataract surgery ${ }^{5}$ that are well known to increase the risk of retinal detachment. In patients with photoreceptor degeneration and hearing loss, as occurs in individuals with Usher syndrome, retinal detachment has a particularly devastating effect upon the quality of life. Here we report the course of three patients with giant retinal tears associated with retinal detachment and underlying photoreceptor dystrophy, the combi-

From: ${ }^{1}$ Vitreoretinal Unit, Moorfields Eye Hospital, London, UK; ${ }^{2}$ Department of Ophthalmology, University of California, San Francisco, California, USA; ${ }^{3}$ Jules Stein Eye Institute, Los Angeles, California, USA.

Correspondence to: Robert Y. Kim, MD, Department of Ophthalmology K301, University of California, San Francisco, 10 Kirkham St, San Francisco, CA 94143-0730, USA. Fax: +1 (415)-476-0336. e-mail: ryk@itsa.ucsf.edu nation of which has been described previously in only two individuals. 6.7

\section{Patient I}

\section{CASE REPORTS}

A 16-year-old girl with congenital total sensorineural deafness was referred in 1987 for evaluation of mildly decreased visual acuities by her optician. Her parents were unrelated with no family history of hearing or visual problems. On examination, she related additional symptoms of nyctalopia and photophobia and was noted to have difficulty walking due to apparent muscle weakness. Best corrected vision in the right eye was $6 / 12\left(-1.75+2.25 \times 105^{\circ}\right)$ and in the left $6 / 60\left(-1.25+2.25 \times 85^{\circ}\right)$. Widespread intraretinal bone spicule pigmentary changes were present in each eye. Goldman perimetry revealed residual central fields of less than $5^{\circ}$ in each eye. Electroretinographic responses were unrecordable. Plasma ornithine and serum phytanic acid levels were normal. VDRL was non-reactive. Muscle biopsy, chromosomal analysis, serum chemistry, electrolytes, and the chest radiograph were normal.

In April 1991, she reported sudden visual loss in the right eye. She was by now wheelchair-bound due to muscle weakness. Visual acuity was counting fingers $(\mathrm{CF})$ in the right eye and $6 / 36$ in the left. A $180^{\circ}$ inferior giant retinal tear with a rolled posterior edge associated with total retinal detachment was present in the right eye. She underwent repair involving pars plana vitreolensectomy and silicone oil exchange. Post-operatively, her retina remained attached but vision was at the perception of light (PL) level. In July 1991, retinal redetachment with rubeosis iridis and hyphaema required membrane dissection and relieving retinectomy. Post-operatively, while vision remained PL and her retina

Eye (1996) 10, 697-700 CC 1996 Royal College of Ophthalmologists 
remained attached, the eye was chronically painful. Retrobulbar alcohol injection in November 1991 failed to relieve her symptoms. Consequently, several weeks later, she underwent enucleation of the then blind right eye.

In March 1993, the patient reported sudden loss of vision in the left eye. Visual acuity was hand motions (HM) due to a total retinal detachment with a giant retinal tear involving the inferior $180^{\circ}$. She underwent lens-sparing pars plana vitrectomy, placement of an encircling scleral buckle, and silicone oil exchange. Visual acuity improved to $\mathrm{CF}$ initially but dropped to HM several months later due to cataract. Cataract extraction was declined. The left eye gradually became blind and painful, leading to enucleation in February 1994. She has since been free of pain.

Macroscopic examination of the left globe revealed an opaque lens, retinal detachment, and silicone oil in the vitreous cavity. Microscopic examination revealed posterior synechiae with rubeosis iridis involving the angle. The retina was shallowly detached in a patchy manner with total loss of inner and outer photoreceptor processes. The choroid was moderately infiltrated with lymphocytes.

\section{Patient 2}

In October 1982, this 11-year-old boy with partial congenital sensorineural hearing loss noticed the sudden onset of 'black vision' in the right eye. His brother, who was 5 years older, also had partial congenital sensorineural hearing loss. His unrelated parents denied any family history of eye or ear problems. When first seen by an optician, vision in the right eye was $6 / 18\left(-6.75+1.25 \times 145^{\circ}\right)$ and in the left $6 / 12\left(-2.75+1.25 \times 75^{\circ}\right)$. (His more highly myopic right eye had always been his weaker eye.) When seen in consultation 3 days later, visual acuity in the right eye was PL and 6/9 in the left. A $165^{\circ}$ giant retinal tear associated with total retinal detachment was present in the right eye. He underwent pars plana vitrectomy, scleral buckle placement and silicone oil exchange. Although the retina was initially attached post-operatively, it redetached 1 month later due to proliferative vitreoretinopathy. $\mathrm{He}$ then underwent repair requiring relieving retinectomy. At this time, retinal cryopexy was applied $360^{\circ}$ to the left eye, in which three flat retinal tears were noted. The retina of the right eye remained attached post-operatively; silicone oil was removed in January 1983. Following cataract extraction 10 months later, his best corrected visual acuity in the right eye was 6/24. In February 1985, he suffered a recurrent retinal detachment associated with vitreous haemorrhage and PL vision. Despite membrane peeling and relieving retinotomy, the retina remained detached. Further surgery was declined. Over the next 9 years, the right eye became hypotonus with band keratopathy and lost perception of light.

In 1990, complaints of photophobia led to evaluation of the left eye. Posterior to retinal cryopexy scars, diffuse but subtle retinal pigmentary changes were evident. Goldman perimetry with a III-4e stimulus was constricted to $20-30^{\circ}$. Electroretinographic responses were unrecordable.

When the patient was last seen in May 1994, vision in the left eye was 6/9 with diffuse intraretinal pigmentary migration, attenuated retinal vessels and peripheral retinal cryopexy scars. His brother was also examined at this time. While diffuse intraretinal bone spicule retinal pigmentary changes were present, no peripheral retinal tears or vitreous base abnormalities were noted. He too had an unrecordable electroretinogram.

\section{Patient 3}

A 15-year-old boy with non-progressive 70 decibel partial hearing loss since birth noted decreased vision in the left eye in 1978 . He denied trauma. A $135^{\circ}$ giant retinal tear with retinal detachment was diagnosed and treated successfully with a scleral buckling procedure. No vitreoretinal pathology was noted in the fellow eye.

Subsequently, the gradual onset of night vision difficulties led to further ophthalmological evaluation in 1981 , at which time visual acuity was $6 / 6$ in the right eye and $6 / 60$ in the left with a correction of $-0.50+0.25 \times 45^{\circ}$ and $-2.50+0.50 \times 120^{\circ}$, respectively. Fundus examination revealed retinal vessel attenuation in both eyes. The right fundus exhibited subtle but widespread pigmentary disturbance at the level of the retinal pigment epithelium extending into the macula. In the left eye, with the exception of retinal scarring and pigmentary changes associated with the scleral buckling procedure, findings were similar to the right eye. Goldman visual fields revealed $10^{\circ}$ central islands. Electroretinographic responses were unrecordable. Final dark-adapted thresholds were elevated by 4 log units. On his last examination in 1991, best corrected visual acuity was $6 / 6$ in the right eye and 6/120 in the left. Fluorescein angiography revealed cystoid macular oedema in the left eye.

This individual's past medical history was remarkable only for a mild heart murmur. He took no medicines for prolonged periods of time. A maternal uncle had decreased vision of unknown aetiology. No other family members had eye problems. The parents were unrelated. VDRL and FTA-Abs were non-reactive. Phytanic acid was normal.

\section{DISCUSSION}

Only two cases of giant retinal tears have been 
described previously in association with underlying retinitis pigmentosa, both of which were associated with congenital hearing loss. ${ }^{6,7}$ In the first, an 18 year-old man with partial congenital hearing loss and retinitis pigmentosa (Usher syndrome type II) suffered bilateral giant retinal tears with retinal detachment in one eye. ${ }^{6}$ The detachment was repaired with vitrectomy, lensectomy and scleral buckling. The fellow eye was treated with retinal cryopexy and an encircling band. One year postoperatively, visual acuity was $20 / 25$ in each eye. The second involved a 14-year-old emmetropic boy with congenital hearing loss (of unspecified severity) and retinitis pigmentosa who presented with a 4 month history of decreased vision in one eye to the PL level. $^{7}$ (At the age of 12 years he had $6 / 6$ vision in each eye.) He was found to have an inoperable retinal detachment arising from a giant retinal tear. In contrast to the first reported case ${ }^{6}$ and our general experience with repairing retinal detachments associated with giant retinal tears, ${ }^{8}$ two of our three patients fared poorly. While patient 1 was not significantly myopic, the more myopic eye of patient 2 suffered the giant retinal tear and retinal detachment. (We do not know the premorbid refractive error of the left eye of patient 3.)

In two of our three cases, underlying retinal dystrophy went undetected prior to the onset of giant retinal tear and retinal detachment. The lack of retinal pigment changes early on led to an 8 year delay in diagnosing retinitis pigmentosa in patient 2 and his brother.

Our patients were all young, hearing-impaired individuals. Usher syndromes comprise a family of photoreceptor dystrophies associated with congenital sensorineural hearing loss. Three types have been described, all of which are inherited in an autosomal recessive manner. Type $\mathrm{I}$ is characterised by progressive photoreceptor degeneration, total congenital sensorineural hearing loss and abnormal vestibular dysfunction. At least three different type I genes have been implicated: one on chromosome 1 (USH1A) ${ }^{9}$ and two on chromosome 11 (USH1B and USH1C). ${ }^{10}$ Type $1 \mathrm{~B}$ Usher syndrome has recently been shown to result from mutations in the gene encoding myosin VIIA, ${ }^{11}$ which in the retina is expressed in the retinal pigment epithelium. ${ }^{12}$ Usher syndrome type II is characterised by progressive photoreceptor degeneration associated with partial, non-progressive congenital sensorineural hearing loss. Linkage analysis indicates that at least two genetically distinct forms of type II Usher syndrome exist, one of which maps to chromosome 1q. ${ }^{13}$ Type III has only recently been defined genetically and is characterised by progressive photoreceptor degeneration with progressive hearing loss and maps to chromosome 3q. ${ }^{14,15}$ While patient 1 exhibits characteristics of Usher syndrome type I, the profound myopathy is not consistent with this diagnosis and suggests alternative diagnoses such as Refsum's disease and mitochondrial myopathies. However, despite extensive investigations, her diagnosis remains unclear. In contrast, patient 2 (and his brother) and patient 3 appear suffer from type II Usher syndrome.

We do not know why the five cases described to date of giant retinal tear associated with underlying retinitis pigmentosa (the patients of Johnston and Gonder, ${ }^{6}$ Edwards et al. $^{7}$ and the three described in this report) have all occurred in young people with congenital sensorineural hearing loss. It is difficult to speculate about mechanisms, given the likely genetic heterogeneity present in this group. While we believe giant retinal tears occur rarely in individuals with retinitis pigmentosa and allied retinal diseases, given the high incidence of bilaterality of non-traumatic giant retinal tears ${ }^{16}$ their occurrence is devastating to those individuals also deprived of their hearing. Vitreous changes have been well documented in retinitis pigmentosa. ${ }^{17.18}$ However, we are unaware of any vitreoretinal interface changes unique to photoreceptor dystrophies associated specifically with sensorineural hearing loss. Although firm epidemiological evidence is unavailable, the literature $^{1-7}$ suggests that the intraretinal migration of retinal pigment epithelium seen clinically in retinal dystrophies ${ }^{19}$ does not protect against retinal detachment and thus differs from the pigmentary changes seen in association with interventional retinopexy procedures.

This work was supported by the Foundation Fighting Blindness (Hunt Valley, MD, USA) and Research to Prevent Blindness (New York, NY, USA). R.Y.K. is the recipient of a Research to Prevent Blindness Career Development Award.

Key words: Giant retinal tear, Hearing loss, Retinal detachment, Retinitis pigmentosa, Usher syndrome.

\section{REFERENCES}

1. Auvert B, Theron H-P, Bescons C. Decollement de retine et retinopathie pigmentaire. Bull Soc Ophtalmol France 1973;73:1159-60.

2. Arakawa T, Nishimura M, Inomata $H$, et al. Pigmentary retinal dystrophy: the statistical studies of 572 cases. Folia Ophthalmol Jpn 1975;26:1036-44.

3. Johnston GP. Retinitis pigmentosa and retina detachment. Retina 1981;1:223-6.

4. Bartkowska-Orlowska M, Orlowski WJ, Pytlarz E. Retinal detachment in eyes with pigmentary retinopathy. Klin oczna 1982;84:251-6.

5. Pruett RC. Retinitis pigmentosa: clinical observations and correlations. Trans Am Ophthalmol Soc 1.983;81:693-735.

6. Johnston ME, Gonder RE. Giant retinal tears, retinal detachment and retinitis pigmentosa. Can J Ophthalmol 1985;20:16-8.

7. Edwards RS, Calder IG, Crews SJ. Retinal detachment 
in retinitis pigmentosa. Trans Ophthalmol Soc UK 1985;104:315-8.

8. Leaver PK, Cooling RJ, Feretis EB, et al. Vitrectomy and fluid/silicone-oil exchange for giant retinal tears: results at six months. Br J Ophthalmol 1984;68:432-8.

9. Kaplan J, Gerber S, Bonneau D, et al. A gene for Usher syndrome type I (USH1A) maps to chromosome 1q. Genomics 1982;14:979-87.

10. Smith RJH, Lee EC, Kimberling WJ, et al. Localisation of two genes for Usher syndrome type I to chromosome 11. Genomics 1992;14:995-1002.

11. Weil D, Blanchard S, Kaplan J, et al. Defective myosin VIIA gene responsible for Usher syndrome type 1B. Nature 1995;374:60-1.

12. Hasson T, Heintzelman MB, Santos-Sacchi J, et al. Expression in cochlea and retina of myosin VIIa, the gene product defective in Usher syndrome type 1B. Proc Natl Acad Sci USA 1995;92:9815-9.

13. Kimberling WJ, Weston MD, Moller C, et al. Gene mapping of Usher syndrome type IIa: localisation of the gene to a $2.1-\mathrm{cM}$ segment on chromosome $1 \mathrm{q} 41$. Am J Hum Genet 1995;56:216-23.

14. Sankila E-M, Pakarinen L, Kaeaeriaeinen $\mathrm{H}$, et al. Assignment of an Usher syndrome type III (USH3) gene to chromosome 3q. Hum Mol Genet 1995;4:93-8.

15. Pakarinen L, Karjalainen S, Simola KOJ, et al. Usher's syndrome type 3 in Finland. Laryngoscope 1995; 105:613-7.

16. Freeman HM. Fellow eyes of giant retinal breaks. Trans Am Ophthalmol Soc 1978;76:343-82.

17. Pruett RC. Retinitis pigmentosa: a biomicroscopical study of vitreous abnormalities. Arch Ophthalmol 1975:93:603-8.

18. Takahashi M, Jalkh A, Hoskins J, et al. Biomicroscopic evaluation and photography in liquefied vitreous in some vitreoretinal disorders. Arch Ophthalmol 1981;199:1555-9.

19. Li ZY, Possin DE, Milam AH. Histopathology of bone spicule pigmentation in retinitis pigmentosa. Ophthalmology 1995;102:805-16. 